\title{
Double Staining of Ginsenosides by Western Blotting Using Anti-ginsenoside Rb1 and Rg1 Monoclonal Antibodies
}

\author{
Noriko Fukuda, Hiroyuki Tanaka, and Yukihiro Shoyama* \\ Graduate School of Pharmaceutical Sciences, Kyushu University, 3-1-1 Maidashi, Higashi-ku, Fukuoka 812-8582, Japan. \\ Received March 19, 2001; accepted June 22, 2001
}

\begin{abstract}
Ginsenosides separated by silica gel TLC blotted to a polyvinylidene difluoride (PVDF) membrane was treated with a $\mathrm{NaIO}_{4}$ solution followed by bovine serum albumin (BSA), resulting in a ginsenoside-BSA conjugate on a PVDF membrane. The blotted bands were stained with anti-ginsenoside Rg1 and Rb1 monoclonal antibodies (MAbs). The newly established double staining with the Western blotting methods was applied for the determination of ginsenosides possessing protopanaxadiol or protopanaxatriol and the number of sugar depending on the stained color and their $R f$ values in the Panax plants.
\end{abstract}

Key words monoclonal antibody (MAb); ginseng; ginsenoside; Western blotting; identification

Ginseng, the crude drug of Panax ginseng is one of the most important Chinese medicines. It has been used to enhance stamina and capacity to cope with fatigue and physical stress, and as a tonic against cancers, disturbances of the central nervous system (memory, learning, and behavior), hypothermia, carbohydrate and lipid metabolism, immune function, the cardiovascular system and radioprotection. ${ }^{1,2)} \mathrm{A}$ group of pharmacologically active components is generally believed to compose the ginsenosides, which consist of protopanaxatriol or protopanaxadiol possessing a dammarane skeleton in their molecules. It is well known that the concentration of ginsenosides varies in the ginseng root or the root extract depending on the method of extraction, subsequent treatment, or even the season of its collection. ${ }^{3)}$ Therefore, standardization of quality is required. For this purpose we previously prepared anti-ginsenoside Rb1 (anti-G-Rb1) monoclonal antibody (MAb) and set up an enzyme-linked immunosorbent assay (ELISA) ${ }^{4)}$ and an immunoaffinity concentration method for one-step isolation of G-Rb1.5) In addition, a new Western blotting using established anti-G-Rb1 MAb was developed. ${ }^{6}$ Although Western blotting is a common assay methodology for substances of high molecular weights, this method has not been employed with small molecules, as direct immunostaining of such compounds on a TLC plate is yet unknown. Therefore, a new method for such small molecular compounds is required. Moreover, if small molecules can be blotted to a membrane, the fixing on it also requires a new methodology. We previously successfully separated the function of small molecular compounds like ginseng saponins into a part of the epitope and the fixing on the membrane. ${ }^{7)}$ More recently, anti-G-Rg1 MAb has been prepared and characterized. ${ }^{8)}$ In our ongoing studies on MAbs against naturally occurring bioactive compounds, ${ }^{9,10)}$ we communicate here a new double staining for ginsenosides by Western blotting using anti-G-Rb1 and G-Rg1 MAbs for the determination of ginsenosides in the crude drug of Panax species.

\section{MATERIALS AND METHODS}

Chemicals and Immunochemicals G-Rb1, -Rc, - Rd, -Re and - Rg1 were purchased from Wako Pure Chemical Ind., Ltd. (Osaka, Japan). Chikusetsusaponin III and IV were donated by Dr. S. Yahara, Faculty of Pharmaceutical Sciences, Kumamoto University. BSA was provided by Pierce (Rockford, IL, U.S.A.). Peroxidase-labeled anti-mouse IgG was provided by Organon Teknika Cappel Products (West Chester, PA, U.S.A.). PVDF membranes (Immobilon-N) were purchased from Millipore Corporation (Bedford, MA, U.S.A.). Glass microfiber filter sheets (GF/A) were purchased from Whatman International Ltd. (Maidstone, England). TLC was performed on precoated Silica gel $60 \mathrm{~F}_{254}$ (Merck, Darmstadt, Germany), and the spots were detected by spraying with $10 \% \mathrm{H}_{2} \mathrm{SO}_{4}$, followed by heating. The crude drug materials of Panax species were purchased from Nakai Koshindo Co., Ltd. (Kobe, Japan). All other chemicals were standard commercial products of analytical grade.

Preparation of Immunoaffinity Column for G-Rb1 Using Anti-G-Rb1 MAb Purified IgG (10 mg) in diluted Bio-Rad Affi Gel Hz coupling buffer was dialyzed against the coupling buffer 2 times. One hundred microliters of $\mathrm{NaIO}_{4}$ solution $(25 \mathrm{mg} / 1.2 \mathrm{ml} \mathrm{H}$ O $)$ was added to the $\mathrm{IgG}$ solution $(1 \mathrm{ml})$ and stirred gently at room temperature in the dark for $1 \mathrm{~h}$. After the reaction, glycerol was added to the reaction mixture for $20 \mathrm{~mm}$ and stirred for $10 \mathrm{~min}$ to inactivate $\mathrm{NaIO}_{4}$, and then dialyzed against the coupling buffer. After the Affi-Gel Hz Hydrazide gel (3 ml) (Bio-Rad, CA, U.S.A.) was washed with the coupling buffer, the buffer was removed. IgG dissolved in the coupling buffer $(5 \mathrm{ml})$ was added to these gels and stirred mildly at room temperature for $24 \mathrm{~h}$. The immunoaffinity gel was packed into a plastic mini-column in volumes of $3 \mathrm{ml}$. Columns were washed with $20 \mathrm{~mm}$ phosphate buffer containing $0.5 \mathrm{M} \mathrm{NaCl}$ (PBS pH 7.0) and stored at $4{ }^{\circ} \mathrm{C}$ in PBS containing $0.02 \%$ sodium azide.

Sample Preparation Dried samples $(50 \mathrm{mg})$ of various types of ginseng were powdered, then extracted with methanol $(5 \mathrm{ml})$ under sonication 5 times, filtered and the combined extract was diluted with $20 \%$ methanol suitable for ELISA and the Western blotting.

Western Blotting and Double Staining Ginsenosides were applied to TLC plates and developed with $n$-BuOHEtOAc- $\mathrm{H}_{2} \mathrm{O}$ (15:1:4, by volume). One TLC plate developed was dried and sprayed with $\mathrm{H}_{2} \mathrm{SO}_{4}$. Another plate was dried and then sprayed with a blotting solution mixture of isopropanol-methanol- $\mathrm{H}_{2} \mathrm{O}(1: 4: 16$, by volume). It was placed on a stainless steel plate, and covered with a PVDF 
membrane sheet. After covering with a glass microfiber filter sheet, the whole was pressed evenly for $50 \mathrm{~s}$ with a $120^{\circ} \mathrm{C}$ hot plate as previously described with modification. ${ }^{10)}$ The PVDF membrane was separated from the plate and dried. The blotted membrane was dipped in water containing $\mathrm{NaIO}_{4}$ with stirring at room temperature for $1 \mathrm{~h}$. After washing with water, $50 \mathrm{~mm}$ carbonate buffer solution $(\mathrm{pH}$ 9.6) containing BSA was added, and stirred for $3 \mathrm{~h}$. The PVDF membrane was washed with TPBS (PBS containing 0.05\% Tween 20) for $5 \mathrm{~min}$ twice, and then washed with water. It was then immersed in anti-G-Rb1 MAb and stirred at room temperature for $1 \mathrm{~h}$. After washing the membrane twice with TPBS and water, 1000 fold dilution of peroxidase-labeled goat antimouse IgG in GPBS (PBS containing $0.2 \%$ gelatin) was added and stirred at room temperature for $1 \mathrm{~h}$. The PVDF membrane was washed twice with TPBS and water, and then exposed for $10 \mathrm{~min}$ at room temperature to $1 \mathrm{mg} / \mathrm{ml}$ freshly prepared of 4-chloro-1-naphthol- $0.03 \% \mathrm{H}_{2} \mathrm{O}_{2}$ in PBS solution.

For the staining by anti-G-Rg1 MAb, the blotted PVDF membrane was treated in the same way as anti-G-Rb1 MAb except that it was exposed to $2 \mathrm{mg} / 10 \mathrm{ml} \mathrm{3}$-amino-9-ethylcarbazole- $0.03 \% \mathrm{H}_{2} \mathrm{O}_{2}$ in acetate buffer $(0.05 \mathrm{M}, \mathrm{pH} 5.0)$ containing $0.5 \mathrm{ml}$ of $N, N$-dimethylformamide.

Separation of G-Rb1 from the Crude Extracts of $\boldsymbol{P}$. japonicus Roots by Immunoaffinity Column Chromatography The extracts of ginseng roots were redissolved in PBS and then filtered through a MILEX-HV filter $(0.45 \mu \mathrm{m}$, Millipore) to remove insoluble portions. The filtrate was loaded on the immunoaffinity column and allowed to stand overnight at $4^{\circ} \mathrm{C}$. The column was washed with the washing buffer solution (PBS $40 \mathrm{ml}$ ), and then eluted with $100 \mathrm{~mm}$ $\mathrm{AcOH}$ buffer containing $0.5 \mathrm{~m} \mathrm{KSCN}$ and $20 \% \mathrm{MeOH}(\mathrm{pH}$ 4.0). The G-Rb1 containing fraction was concentrated and surveyed by TLC developed with $n$-BuOH-AcOEt- $\mathrm{H}_{2} \mathrm{O}$ ( $15: 1: 4$, by volume), followed by Western blotting.

Separation of an unknown ginsenoside from the crude extractions of Panax japonicus root by an immunoaffinity column chromatography was done by the same way as described above.

\section{RESULTS AND DISCUSSION}

Western Blotting of Ginsenosides Using Anti-G-Rg1 MAb The blotted PVDF membrane was treated with $\mathrm{NaIO}_{4}$ solution. This reaction enhanced the fixing of ginsenosides via ginsenoside-BSA conjugates on the membrane. Incubated in the absence of $\mathrm{NaIO}_{4}$, the membrane was essentially free of staining for ginsenosides (data not shown).

Figure 1 shows the $\mathrm{H}_{2} \mathrm{SO}_{4}$ staining (A), the Western blotting of ginsenoside standards and the crude extract of various Panax species root using anti-G-Rg1 MAb (B). The Western blotting method was considerably more sensitive than the $\mathrm{H}_{2} \mathrm{SO}_{4}$ staining. Lanes 1 to 6 show the bands of ginsenosides, G-Rb1, -Rc, -Rd, -Re and -Rg1 which all possess a dammarane skeleton in a molecule, and chikusetsusaponin IV which has an oleanane aglycon, respectively. The $\mathrm{H}_{2} \mathrm{SO}_{4}$ staining detected all standard compounds including chikusetsusaponin IV. Furthermore, many bands other than those of ginsenosides appeared in the crude extract. The Western blotting, however, indicated only limited staining of G-Re and G-
Rg1 as shown in Fig. 1B lanes 4 and 5, respectively, these bands were detected in the crude extracts. Although the cross-reactivity of G-Re against anti-G-Rg1 MAb is $3.3 \%$ as reported previously, ${ }^{8)}$ it can be stained by anti-G-Rg1 MAb. This phenomenon is similar to that of anti-G-Rb1 MAb which detected G-Rc and G-Rd strongly although their crossreactivities were 0.024 , and also weakly detected G-Re and G-Rg1 of which cross-reactivities were under $0.005 \%{ }^{4}{ }^{4} \mathrm{We}$ suggested that an aglycon, protopanaxadiol and part of the sugars may be of importance to the immunization and may function as an epitope for the structure of ginsenosides. It is also suggested that the specific reactivity of sugar moiety in the ginsenoside molecule against anti-G-Rg1 MAb may be modified by the $\mathrm{NaIO}_{4}$ treatment of ginsenosides on the membrane causing G-Re to be detectable by Western blotting.

Double Staining of Ginsenosides Using Anti-G-Rb1 and Anti-G-Rg1 MAb When the mixture of anti-G-Rg1 and -G-Rb1 MAbs, and the pair of substrates were tested for the staining of ginsenosides, all ginsenosides, G-Rg1, -Re, -Rd, -Rc and -Rb1 were stained a blue color as shown in Fig. 2, although a red stain for G-Rg1 was expected because 3-amino-9-ethylcarbazole was used as a substrate. It is suggested that the sensitivities of substrate between 3-amino-9ethylcarbazole and 4-chloro-1-naphthol might be different. Therefore, we performed successive staining of the membrane using anti-G-Rg1 and then anti-G-Rb1. Finally, we succeeded in the double staining of ginsenosides indicating that G-Rg1 and G-Re were stained red and the others blue as indicated in Fig. 3. From this result both antibodies can distinguish individual aglycons, protopanaxatriol and protopanaxadiol. For this application the crude extracts of various Panax species were analyzed by the newly developed double staining system. Major ginsenosides can be determined clearly by the double staining method as indicated in Fig. 4. Three new bands stained by anti-G-Rg1 MAb were found as indicated by arrows, although they could not be detected by the staining with anti-G-Rb1 MAb as previously reported. ${ }^{6}$ It is evident that these bands related to ginsenosides having protopanaxatriol in a molecule. A search of previous literature $^{11)}$ allowed us to conclude that the three bands were G-Rh1, G-Rf and 20-gluco-G-Rf which were mono-, di- and triglycoside, respectively depending upon their $R f$ values. This makes it possible to suggest the structures of ginsenosides by judging from their band color and the $R f$ value reflected from the sugar number.

The substitution pattern of the sugars is of importance. The sugar moiety at C-3 position was necessary for staining, but that at C-6 position inhibited staining using anti-G-Rb1 MAb.

In contrast, this investigation ruled out that the sugar conjugation at C-3 position completely inhibited staining because none of the protopanaxadiol series like G-Rb1, G-Rc and G-Re could stained by anti-G-Rg1 MAb. However, the substitution of $\mathrm{C}-20$ position is not related to the staining since 20 -gluco-G-Rf was detected. From these results, the most important position is C-6 combined with sugar moiety for staining. It is summarized that the areas around A and B rings in ginsenoside function as a part of the epitope, but $\mathrm{C}$ 20 position does not.

Isolation and Determination of Ginsenosides by Im- 
A

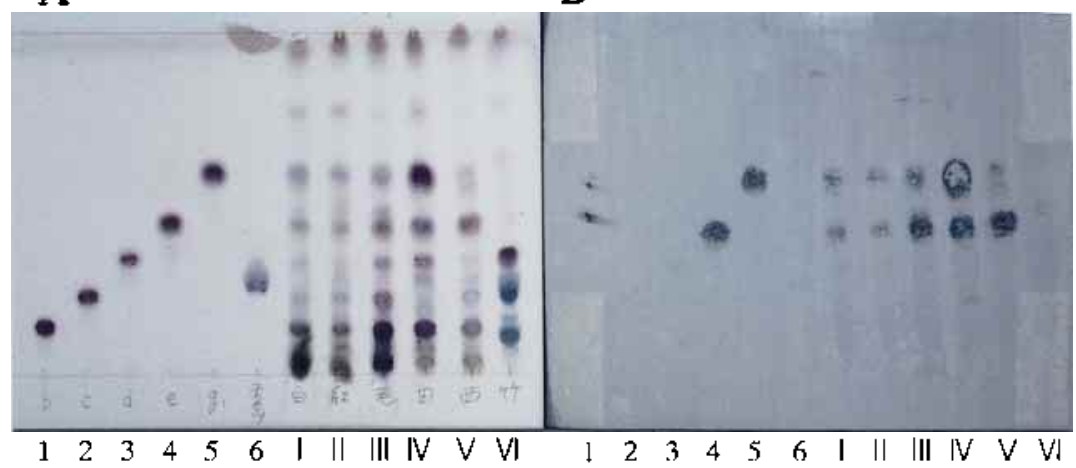

Fig. 1. TLC (A: $\mathrm{H}_{2} \mathrm{SO}_{4}$ Staining) and Western Blotting (B) Profiles of Ginsenoside Standards and the Crude Extract of Various Panax Species Roots Using Anti-G-Rg1 MAb

Lanes 1, 2, 3, 4, 5 and 6 indicate G-Rb1, G-Rc, G-Rd, G-Re, G-Rg1, and chikusetsusaponin IV (3 $\mu$ g), respectively. Lanes I, II, III, IV, V, and VI are white ginseng, red ginseng, fibrous ginseng, P. notoginseng, P. quinquefolium, and P. japonicus $(60 \mu \mathrm{g})$, respectively.

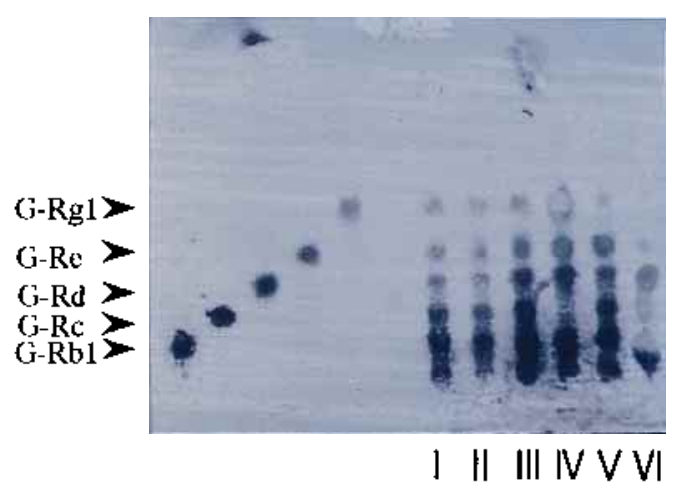

Fig. 2. Western Blotting Profiles of Ginsenoside Standards and the Crude Extract of Various Panax Species Root Using Anti-G-Rg1, G-Rb1 MAb Mixture

Lanes I, II, III, IV, V, and VI indicate white ginseng, red ginseng, fibrous ginseng, $P$. notoginseng, P. quinquefolium, and $P$. japonicus $(60 \mu \mathrm{g})$, respectively.

A

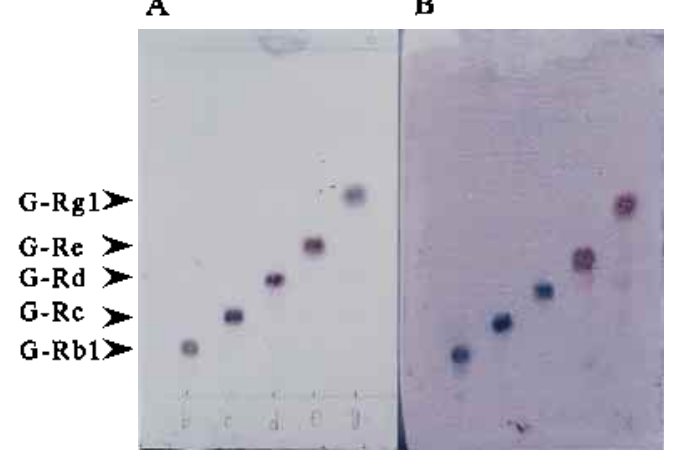

Fig. 3. Double Staining of Ginsenosides

A, $\mathrm{H}_{2} \mathrm{SO}_{4}$ staining; B, Western blotting. Red and blue colors were stained by anti-G$\mathrm{Rg} 1 \mathrm{MAb}$ and $\mathrm{Rb} 1 \mathrm{MAb}$, respectively.
A

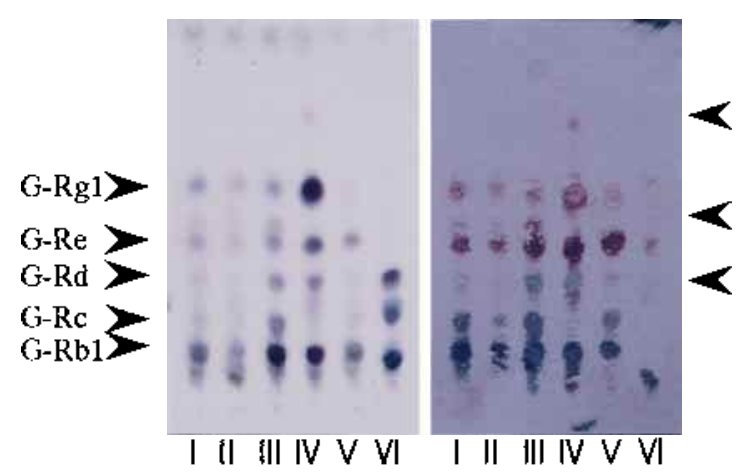

Fig. 4. Double Staining of Various Panax Samples

A, $\mathrm{H}_{2} \mathrm{SO}_{4}$ staining; B, Western blotting. Lanes I, II, III, IV, V, and VI indicate white ginseng, red ginseng, fibrous ginseng, $P$. notoginseng, P. quinquefolium, and $P$. japonicus $(60 \mu \mathrm{g})$, respectively.
A

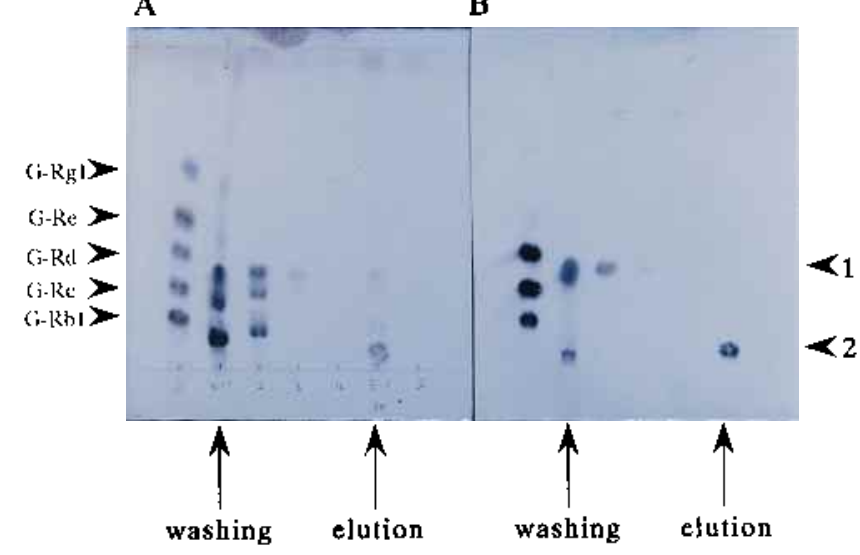

Fig. 5. TLC (A: $\mathrm{H}_{2} \mathrm{SO}_{4}$ Staining) and Western Blotting (B) Profiles of Fractions Separated by Immunoaffinity Concentrations of $P$. japonicus Extract

After the column was washed with PBS $(40 \mathrm{ml})$, bound ginsenosides were eluted with $100 \mathrm{~mm} \mathrm{AcOH}$ buffer containing $0.5 \mathrm{M} \mathrm{KSCN}$ and $20 \% \mathrm{MeOH}(\mathrm{pH} 4.0)$. The washed and eluted fractions were assayed. 


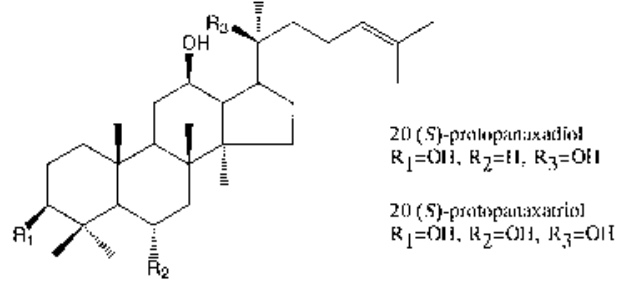

\begin{tabular}{|c|c|c|c|c|}
\hline Compronsult & Aylyam & $\mathbf{R}_{1}$ & $\mathrm{R}_{2}$ & $\mathrm{R}_{3}$ \\
\hline Giscrnksids Rh1 & Prutopenthax:ltrial & $\mathrm{OH}$ & QGe & $\mathrm{OH}$ \\
\hline Girsensilicre Rgy & & CHI & $|0-6| c$ & $-(0-6) \mid c$ \\
\hline Gitrseruswidle RI & & DH & -0 Glétre & $\mathrm{OH}$ \\
\hline Ginsenusilde Ro & & $\mathrm{OH}$ & $-0 . G c^{2} \mathrm{~B} h a$ & $.0-G k^{2}$ \\
\hline 20-Glueoginsenoside Re & & OE & $-0-6 j\left|L^{2}-G\right|<$ & $.00 \mathrm{k}$ \\
\hline Ginncrusside R.L & 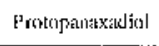 & $-0-\mathrm{Cile}_{2}^{2}-\mathrm{Gl}$ & נt & $-0 \cdot \mathrm{Gic}$ \\
\hline Ginnentoside $\mathrm{Re}$ & & $-0-G] \mathrm{e}^{2} \mathrm{G} \mid \mathrm{r}$ & $\mathrm{H}$ & -G-Gle:Ala \\
\hline Gilmstioside Rly] & & $-0-[\mathrm{G}] \mathrm{c}^{2} \mathrm{Gle}$ & H & 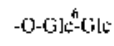 \\
\hline 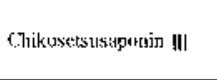 & & $\begin{array}{r}0 . \mathrm{Gle} \mathrm{x}_{\mathrm{yl}} \\
\underbrace{}_{\mathrm{Gle}}\end{array}$ & $\mathrm{H}$ & $\mathrm{OH}$ \\
\hline Clikusecsu.sapaniny & & 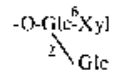 & H & $-0-\mathrm{Gi} \mathrm{c}^{6} \mathrm{Gle}$ \\
\hline
\end{tabular}

Fig. 6. Saponins from Panax spp. Root

munoaffinity Concentration Using Anti-G-Rb1 MAb We earlier prepared an immunoaffinity column combined with anti-G-Rb1 MAb and concentrated G-Rb1 in the crude extract of $P$. ginseng by one-step purification. ${ }^{5)}$ In this case $\mathrm{G}-\mathrm{Rb} 1$ was eluted by an elution buffer together with malonyl-G-Rb1 because it had similar cross-reactivity to that of $\mathrm{G}-\mathrm{Rb} 1$. This suggested that some new compounds having the same cross-reactivity could be eluted with G-Rb1. To search for unknown compounds in P. japonicus, the crude extract of its root was affinity concentrated by the affinity gels conjugated with anti-G-Rb1 MAb. After washing, the column was eluted with the elution buffer to give 2 compounds, bands 1 and 2 indicating a blue color that means a protopanaxadiol skeleton detected by Western blotting using anti-G-Rb1 MAb as shown in Fig. 5. Band 1 had an $R f$ value close to that of G$\mathrm{Rd}$ suggesting that this band has three sugar moieties in a molecule. Judging from the survey of ginsenosides by previous papers ${ }^{12)}$ we determined that band 1 is chikusetsusaponin III that has three sugars, xylose and 2 glucose in a molecule as indicated in Fig. 6. Band 2 is more polar than G-Rb1, indicating that it exhibits more than four sugars or may be malonyl-G-Rb1. Although band 2 was treated with a mild alkaline solution, no change occurred. From these results we confirmed that this band is 20-O-gentiobiosyl-chikusetsusaponin III (chikusetsusaponin VI) and has five sugars in a molecule in good agreement with the $R f$ value previously reported. ${ }^{13)}$ Finally, these two compounds were identified by comparison with the authentic samples.

\section{CONCLUSION}

We established a new double staining method for ginseno- sides using anti-G-Rb1 and -G-Rg1 MAbs. This system enhanced the separate staining of ginsenosides having protopanaxatriol or protopanaxadiol in a molecule as an aglycon. The $R f$ value of ginsenosides roughly suggests the number of sugars attached to the aglycon. Therefore, both analyses make it possible to suggest which aglycon is attached and how many sugars are combined leading to the structure of ginsenosides. In fact, three kinds of ginsenosides possessing protopanaxadiol, G-Rh1, G-Rf and 20-O-gluco-G-Rf in $P$. ginseng root were determined by their coloring and $R f$ value compared with the structures reported previously. ${ }^{11)}$ We also identified two dammarane saponins having protopanaxadiol, chikusetsusaponin III and chikusetsusaponin VI in P. japonicus root after immunoaffinity concentration using an affinity column conjugated with anti-G-Rb1 MAb. This newly established methodology could make it possible to survey the natural resources of ginsenosides and to quickly determine their structures.

This is apparently the first report of the double staining of ginsenosides and its application. To date it has been difficult to detect small molecular compounds by Western blotting and/or immunocytolocalization, and the methodology described here may open a wide field of comparable studies with other families of carbohydrates containing compounds of low molecular weight, such as saponins, glycosides, glucuronides and aminosugar conjugates.

Acknowledgements The authors thank Dr. S. Yahara, Faculty of Pharmaceutical Sciences, Kumamoto University for his helpful discussion and donation of chikusetsusaponin III \& VI. This research was supported by a Grant-in-Aid for Scientific Research, from The Ministry of Education, Science, Sports, Culture, and Technology, the Shorai Foundation for Science and Technology.

\section{REFERENCES}

1) Tanaka Y., Akagi K., "Metabolism and Disease," Vol. 29, ed. by Okuda T., Kimura M., Miyamoto A., Wada H., Nakayama Shoten, Tokyo, 1992, supplementary issue, pp. 423-429.

2) Shibata S., J. Trad Sin-Jpn. Med., 3, 62-69 (1982).

3) Kim S. K., Sakamoto I., Morimoto K., Sakata M., Yamasaki K., Tanaka O., Planta Medica, 42, 181-186 (1981).

4) Tanaka H., Fukuda N., Shoyama Y., Cytotechnology, 29, 115-120 (1999).

5) Fukuda N., Tanaka H., Shoyama Y., J. Nat. Prod., 63, 283-285 (2000).

6) Fukuda N., Tanaka H., Shoyama Y., Biol. Pharm. Bull., 22, 219-220 (1999).

7) Tanaka H., Putalun W., Tsuzaki C., Shoyama Y., FEBS Lett., 404, 279-282 (1997).

8) Fukuda N., Tanaka H., Shoyama Y., Cytotechnology, 34, 197-204 (2000).

9) Shoyama Y., Yakugaku Zasshi, 120, 749-765 (2000).

10) Shoyama Y., Tanaka H., Fukuda N., Shan S. J., Muraoka K., Res. Adv. in Phytochem., 1, 83-104 (2000).

11) Shoji J., "Recent Advances in Ginseng Studies," ed. by Shibata S., Ohtuka Y., Saito H., Hirokawa Publications, Tokyo, 1990, pp. 11-31.

12) Morita T., Tanaka O., Kohda H., Chem. Pharm. Bull., 33, 3852-3858 (1985).

13) Kohda H., Tanaka S., Yamaoka Y., Izumi H., Nuno M., Isoda S., Gotoh K., Watanabe T., Katsuki S., Satake M., Chem. Pharm. Bull., 39, 1588-1590 (1991). 